\title{
Spatial resolution limits for the localization of noise sources using direct sound mapping
}

\author{
Comesana, D. Fernandez; Holland, K. R.; Fernandez Grande, Efren
}

Published in:

Journal of Sound and Vibration

Link to article, DOI:

10.1016/j.jsv.2016.04.010

Publication date:

2016

Document Version

Peer reviewed version

Link back to DTU Orbit

Citation (APA):

Comesana, D. F., Holland, K. R., \& Fernandez Grande, E. (2016). Spatial resolution limits for the localization of noise sources using direct sound mapping. Journal of Sound and Vibration, 375, 53-62.

https://doi.org/10.1016/j.jsv.2016.04.010

\section{General rights}

Copyright and moral rights for the publications made accessible in the public portal are retained by the authors and/or other copyright owners and it is a condition of accessing publications that users recognise and abide by the legal requirements associated with these rights.

- Users may download and print one copy of any publication from the public portal for the purpose of private study or research.

- You may not further distribute the material or use it for any profit-making activity or commercial gain

- You may freely distribute the URL identifying the publication in the public portal 


\title{
Spatial resolution limits for the localization of noise sources using direct sound mapping
}

\author{
D. Fernandez Comesaña ${ }^{\text {a,* }}$, K.R. Holland ${ }^{b}$, E. Fernandez-Grande ${ }^{c}$ \\ a Microflown Technologies, 6824 BV Arnhem, The Netherlands \\ ${ }^{\mathrm{b}}$ Institute of Sound and Vibration Research, University of Southampton, Southampton SO17 1BJ, UK \\ ${ }^{\text {c } D T U ~-~ T e c h n i c a l ~ U n i v e r s i t y ~ o f ~ D e n m a r k, ~ D K-2800 ~ K g s . ~ L y n g b y, ~ D e n m a r k ~}$
}

\section{A R T I C L E I N F O}

\section{Article history:}

Received 15 January 2016

Received in revised form

16 March 2016

Accepted 11 April 2016

Handling Editor: P. Joseph

\section{Keywords:}

Direct sound mapping

Spatial resolution

Source localization

\begin{abstract}
A B S T R A C T
One of the main challenges arising from noise and vibration problems is how to identify the areas of a device, machine or structure that produce significant acoustic excitation, i.e. the localization of main noise sources. The direct visualization of sound, in particular sound intensity, has extensively been used for many years to locate sound sources. However, it is not yet well defined when two sources should be regarded as resolved by means of direct sound mapping. This paper derives the limits of the direct representation of sound pressure, particle velocity and sound intensity by exploring the relationship between spatial resolution, noise level and geometry. The proposed expressions are validated via simulations and experiments. It is shown that particle velocity mapping yields better results for identifying closely spaced sound sources than sound pressure or sound intensity, especially in the acoustic near-field.
\end{abstract}

(c) 2016 Elsevier Ltd. All rights reserved.

\section{Introduction}

Over the last decades there has been dramatic progress in the development of acoustic imaging techniques [1-3]. The transformation of sound into something visual is considered key to understanding a wide variety of problems. Many techniques and apparatus have been proposed over the years, most with a common goal: localize where sound originates. Although noise can be the result of a complex chain of events, finding the areas of a machine or structure that create a significant acoustic excitation is a good starting point for applying appropriate noise control measures.

A large number of methods have been developed for pressure microphone arrays [4]. Near-field acoustic holography [5], acoustic beamforming [6] and various inverse methods [7] offer different approaches to localize, and ultimately quantify, the sources of noise. However, pressure-based techniques often encounter difficulties adapting from controlled experiments to industrial applications [8]. In many cases the presence of various critical factors such as source dimensions, room reverberation or noise produced by surrounding machinery may increase estimation error, ultimately limiting the ability to resolve the assessed sound sources accurately $[9,10]$.

The direct visualization of sound, in particular sound intensity, has been used extensively since the 1980s to locate, quantify and rank sound sources [4]. Ever since the introduction of sound intensity probes a series of standardized measurement methods have been available for performing the in situ characterization of complex sound sources. Despite its

\footnotetext{
* Corresponding author.

E-mail addresses: fernandez@microflown.com (D. Fernandez Comesaña), krh@isvr.soton.ac.uk (K.R. Holland), efg@elektro.dtu.dk (E. Fernandez-Grande).
} 
simplicity, the direct representation of sound intensity has proven very useful for a wide range of practical cases. Although there is an extensive amount of literature that covers the foundations of sound intensity for noise quantification [11,12], a detailed description of the spatial resolution limit for direct sound intensity mapping is still not yet defined.

In recent years, the introduction of new mapping techniques which enable the rapid visualization of spatial sound distributions [13-15] has been applied to multiple industrial problems [16-18], increasing the interest in exploring the foundations of the direct representation of sound. This paper evaluates several spatial resolution criteria and introduces a novel model to establish the resolution limits of direct sound pressure, acoustic particle velocity and sound intensity mapping.

\section{Resolution criteria}

Several criteria can be used to describe when two sources should be regarded as resolved. Since resolution is not unambiguously defined, various interpretations have been proposed during the last two centuries, most of them originally introduced in the field of optics [19-21]. The natural analogy between acoustics and optics is explored in this section to derive the most popular resolution criteria using a well-defined technique such as acoustic beamforming. The assumptions described in this section are hence independent of the underlying theory of direct sound mapping. However, the results obtained will be used to understand what can be considered as resolvable by applying an objective quantification of spatial resolution to the output signal.

The derivation that follows considers the limits of a transducer array sensing incoherent sound waves using acoustic beamforming [22,23]. Considering a continuous line array located along the $x$-axis of size $D$, when a wave front with a wavenumber vector $\mathbf{k}=\left[k_{x}, k_{y}, k_{z}\right]$ impinges upon the array it produces an output proportional to the spatial integral over the aperture [7]

$$
W(\mathbf{k})=\int_{-D / 2}^{D / 2} b(\mathbf{r}) \Phi(\mathbf{r}, \mathbf{k}) \mathrm{d} x,
$$

where $b(\mathbf{r})$ represents a spatial weighting function and $\Phi(\mathbf{r}, \mathbf{k})$ denotes the quantity being measured along the array at position $\mathbf{r}$. For the simple case of a plane wave travelling towards a uniform linear array of sound pressure sensors located at $\mathbf{r}=[x, 0,0]$

$$
\Phi(\mathbf{r}, \mathbf{k})=e^{j(\mathbf{k} \cdot \mathbf{r})}=e^{j k_{x} x} .
$$

Using a uniform weighting $b(\mathbf{r})=1 / D$, and combining Eqs. (1) and (2) the array output produces an interference pattern that varies following a sinc function such as

$$
W(\mathbf{k})=\frac{\sin \left(k_{x} D / 2\right)}{k_{x} D / 2} .
$$

The array pattern can be steered towards a certain direction $\mathbf{k}_{0}$ by evaluating $W\left(\mathbf{k}-\mathbf{k}_{0}\right)$. As a result, the combination of signals captured within the aperture will yield the highest output when it is steered towards the sound direction of arrival.

The definition of the array output presented in Eq. (3) is assessed below using the main resolution principles commonly used: the Rayleigh and the Full Width at Half Maximum (FWHM) criteria. The main differences between these two methods are then studied using the Valley to Peak ratio ( $V / P$ ratio). The derivations that follow are focused on the particular case when two uncorrelated wavefronts travelling in two slightly different directions are sensed by a uniform linear aperture as two different sources.

\subsection{Rayleigh limit}

One classical definition of resolution is the Rayleigh criterion [24]. It is assumed that a pair of incoherent plane waves can be resolved if the shifted peak of the aperture smoothing function $W\left(\mathbf{k}-\mathbf{k}_{1}\right)$ falls on the first zero when pointing towards the other direction of propagation $W\left(\mathbf{k}-\mathbf{k}_{2}\right)$. Therefore, the Rayleigh resolution is equal to the smallest wavenumber that produces a zero in the array pattern $W(\mathbf{k})$, i.e.

$$
\min \left(k_{x}\right)=k \sin \left(\theta_{\min }\right)=\frac{2 \pi}{D} .
$$

The following relationship can be established by focusing the array on a source plane located at a distance $d$

$$
\sin \left(\theta_{\min }\right)=\frac{R_{R}}{\sqrt{R_{R}^{2}+d^{2}}}
$$

where $R_{R}$ denotes the minimum resolvable distance between sources according to the Rayleigh criterion. Given that 
$k=2 \pi / \lambda$, the combination of Eqs. (4) and (5) yields

$$
R_{R}=\frac{d \lambda}{\sqrt{D^{2}-\lambda^{2}}} .
$$

In the particular case when the aperture size is much larger than the acoustic wavelength $(D>>\lambda)$, the above expression leads to the classical definition of the Rayleigh resolution limit, i.e.

$$
R_{R} \approx \frac{d}{D} \lambda
$$

\subsection{Full Width at Half Maximum}

In 1927, Houston proposed the use of the Full Width at Half Maximum (FWHM) to quantify resolution [25] based on the response of the aperture to a single source, the so-called Point Spread Function (PSF). This principle is also known as the $3 \mathrm{~dB}$ bandwidth criterion. The FWHM limit is widely used both in optics and acoustics because, unlike Rayleigh criterion, it is also suitable for PSFs that do not fall off to zero. Considering the aperture introduced above, the FWHM can be derived from Eq. (3) by imposing the following initial condition:

$$
|W(\mathbf{k})|^{2}=\frac{\sin ^{2}(\alpha)}{\alpha^{2}}=0.5,
$$

where $\alpha=k \sin \left(\theta_{\text {FWHM }}\right) D / 2$ which can be shown numerically as $\alpha \approx 1.39$ for a uniform linear array. Focusing the array on a plane located at a distance $d$, the FWHM resolution angle $\theta_{\mathrm{FWHM}}$ and resolution distance $R_{\mathrm{FWHM}}$ are related as follows:

$$
\sin \left(\theta_{\mathrm{FWHM}}\right)=\frac{R_{\mathrm{FWHM}}}{2\left(R_{\mathrm{FWHM}}^{2} / 4+d^{2}\right)^{1 / 2}} .
$$

The combination of Eqs. (8) and (9) defines the resolution distance according to the FWHM criterion

$$
R_{\mathrm{FWHM}}=\frac{4 \alpha d}{\sqrt{D^{2} k^{2}-4 \alpha^{2}}} .
$$

When the aperture size is much larger than the wavelength $(D>>\lambda)$ the above expression can be simplified such as

$$
R_{\mathrm{FWHM}} \approx 0.89 \frac{d}{D} \lambda \text {. }
$$

\subsection{Valley to Peak ratio}

The Valley to Peak ratio ( $V / P$ ratio) is a quantity linked to resolution that measures the extent of separation between two peaks $[26,27]$. It can be computed from the array output as the ratio of the minimum observed between two sources of equal strength to the maximum achieved in front of them. The $V / P$ ratio was proposed complementary to resolution and can be used to quantify the quality of the different criteria described above.

Fig. 1 shows an example of the array output in the presence of two uncorrelated sound sources when they are separated by the minimum distance suggested by Rayleigh (left) and the FWHM criterion (right). The corresponding $V / P$ ratio, hereby denoted as $\beta$, can be computed numerically yielding $\beta \approx 0.81$ for the Rayleigh criterion and $\beta \approx 0.98$ for the FWHM method.

(a)

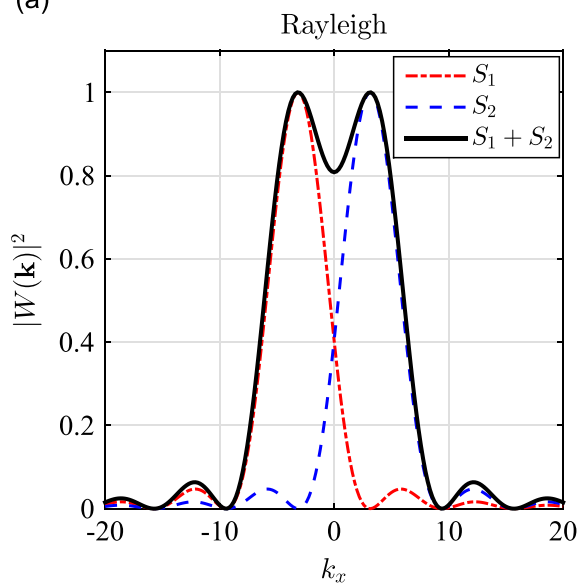

(b)

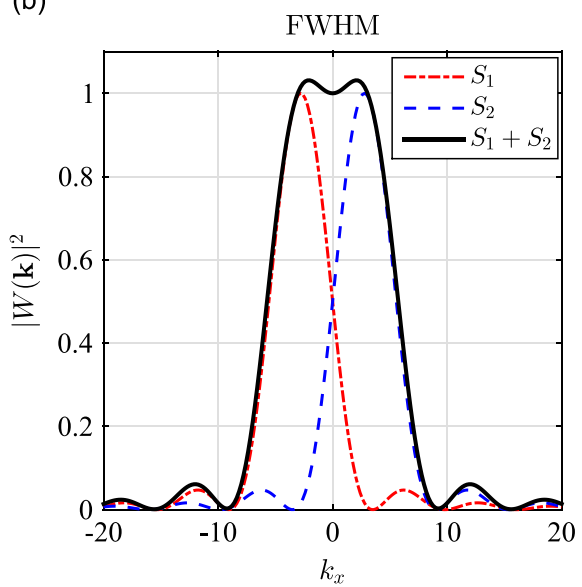

Fig. 1. Comparison of Rayleigh and FWHM resolution criteria. 


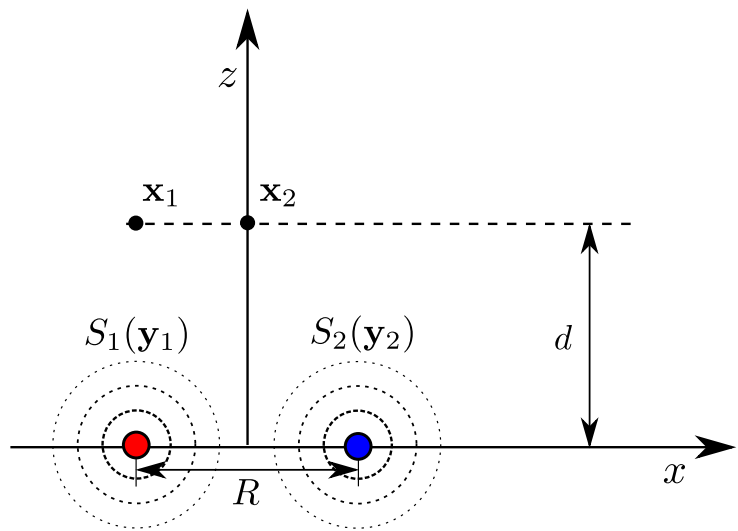

Fig. 2. Sketch of the problem assessed.

Recalling the definition of spatial resolution, i.e. the capability of resolving two sources, the $V / P$ ratio can be seen as a useful link between different resolution criteria. The importance of this parameter will become apparent in the following section since it is used to formulate the initial condition necessary for the derivation of the limits of direct sound mapping methods. As shown, although in both results the sources can be distinguished, the FWHM resolution criterion is less clear. The same conclusion can be inferred from the comparison of Eqs. (7) and (11). In the following derivation the Rayleigh criterion is used as reference due to its robustness.

\section{Direct sound mapping resolution limits}

The spatial resolution of a sound localization method determines the ability to distinguish two closely spaced noise sources. Due to the analogy with classical optics it is commonly assumed that the spatial resolution of most sound localization methods is limited by the wavelength of radiation [8]. However, in acoustic near-fields, it is possible to overcome the spatial resolution limit via the inclusion of evanescent waves $[28,29,5]$.

A generalized solution for the resolution limit of direct sound mapping can be formulated regardless of the acoustic quantity being represented. This section examines the common derivation that can be applied to study the limits of sound pressure, particle velocity or sound intensity mapping in the presence of two uncorrelated point sources. A sketch of the problem addressed is illustrated in Fig. 2. Although the result obtained would vary depending on the nature of the real source, the assumptions used below are similar to those introduced to derive the limitations of other techniques such as beamforming.

Let us define two sound sources $S_{1}\left(\mathbf{y}_{1}\right)$ and $S_{2}\left(\mathbf{y}_{2}\right)$ located at $\mathbf{y}_{1}=[-R / 2,0,0]$ and $\mathbf{y}_{2}=[R / 2,0,0]$, where $R$ is the minimum separation distance at which the sources can be resolved. The sound field is then studied through measurements recorded at plane $z=d$. Considering the definition of the $V / P$ ratio presented above, two measurement locations are of particular interest: the local maxima perceived in front of the sources and the minimum formed between them. Consequently, the sound field perceived at $\mathbf{x}_{1}=[-R / 2,0, d]$ and $\mathbf{x}_{2}=[0,0, d]$ is hereby studied. The $V / P$ ratio $\beta$ can be used as the initial condition of the derivation, such as

$$
\left|\Phi\left(\mathbf{x}_{1}, \omega\right)\right|^{2} \beta=\left|\Phi\left(\mathbf{x}_{2}, \omega\right)\right|^{2},
$$

where $|\Phi(\mathbf{x}, \omega)|^{2}$ is the spectral power output of a sensor at frequency $\omega$. Suppose the sound field created by a source can be described by the product of an arbitrary function $A(\omega)$ associated with the source excitation input and a spatial propagation function $h(\mathbf{x} \mid \mathbf{y})$ which relates the noise emitted at $\mathbf{y}$ to the sound field perceived at $\mathbf{x}$. The squared amplitude of $\Phi(\mathbf{x}, \omega)$ in the presence of two uncorrelated sound sources can then be described by the following model:

$$
|\Phi(\mathbf{x}, \omega)|^{2}=A_{1}^{2}(\omega) h^{2}\left(\mathbf{x} \mid \mathbf{y}_{1}\right)+A_{2}^{2}(\omega) h^{2}\left(\mathbf{x} \mid \mathbf{y}_{2}\right)+\sigma_{n}^{2},
$$

where $\sigma_{n}^{2}$ represents the variance of the unwanted noise captured by the measuring system.

For the problem addressed, it can be assumed that $h\left(\mathbf{x}_{2} \mid \mathbf{y}_{1}\right)=h\left(\mathbf{x}_{2} \mid \mathbf{y}_{2}\right)$ and that the sound sources have equal excitation power $A_{1}(\omega)=A_{2}(\omega)=A$. Combining Eqs. (12) and (13) then yields

$$
\left(h^{2}\left(\mathbf{x}_{1} \mid \mathbf{y}_{1}\right)+h^{2}\left(\mathbf{x}_{1} \mid \mathbf{y}_{2}\right)+\sigma_{n}^{2} / A^{2}\right) \beta=2 h^{2}\left(\mathbf{x}_{2} \mid \mathbf{y}_{1}\right)+\sigma_{n}^{2} / A^{2} .
$$

When measuring in front of a source, the signal-to-noise ratio can be defined as the signal power received from the sound source compared to the unwanted noise captured by the system, i.e.

$$
\mathrm{SNR}=\left(\frac{A_{1}(\omega) h\left(\mathbf{x}_{1} \mid \mathbf{y}_{1}\right)}{\sigma_{n}}\right)^{2}=\left(\frac{A_{2}(\omega) h\left(\mathbf{x}_{2} \mid \mathbf{y}_{2}\right)}{\sigma_{n}}\right)^{2} .
$$


The combination of Eqs. (14) and (15) then leads to

$$
\left(\beta+\frac{\beta-1}{\mathrm{SNR}}\right) h^{2}\left(\mathbf{x}_{1} \mid \mathbf{y}_{1}\right)+\beta h^{2}\left(\mathbf{x}_{1} \mid \mathbf{y}_{2}\right)-2 h^{2}\left(\mathbf{x}_{2} \mid \mathbf{y}_{1}\right)=0 .
$$

This expression establishes common ground that is suitable for deriving the direct mapping resolution limits of any arbitrary measured quantity $\Phi(\mathbf{x}, \omega)$. In the following sections Eq. (16) is evaluated in terms of sound pressure, normal particle velocity and sound intensity. For the sake of clarity, the resolution of the different mapping methods is hereby denoted as $R_{p}$, $R_{u}$ and $R_{I}$ to indicate the minimum resolvable distance of the corresponding mapping method, respectively.

\subsection{Sound pressure}

The sound pressure perceived at a point $\mathbf{x}$ generated by a harmonic monopole source situated at $\mathbf{y}$ is defined as [11]

$$
p(\mathbf{x} \mid \mathbf{y}, t)=\frac{\mathrm{j} \omega \rho Q}{4 \pi r} e^{\mathrm{j}(\omega t-k r)},
$$

where $Q$ is the volume velocity of the source, $\rho$ is the air density, $k$ is the wavenumber, $\omega$ is the angular frequency and $r$ is the distance between the two points $\|\mathbf{x}-\mathbf{y}\|$. In order to apply the derivation proposed above, it is first necessary to define the sound pressure auto-power spectrum, i.e.

$$
S_{p p}(\mathbf{x} \mid \mathbf{y}, \omega)=P(\mathbf{x} \mid \mathbf{y}, \omega) P^{*}(\mathbf{x} \mid \mathbf{y}, \omega)=\left(\frac{\omega \rho|Q|}{4 \pi}\right)^{2}\left(\frac{1}{r}\right)^{2}=A^{2}(\omega) h^{2}(\mathbf{x} \mid \mathbf{y}),
$$

where $P(\mathbf{x} \mid \mathbf{y}, \omega)$ is the Fourier transform of $p(\mathbf{x} \mid \mathbf{y}, t)$. For the geometry studied and given Eq. (18), the spatial propagation functions required to solve Eq. (16) become

$$
\left\{\begin{array}{l}
h^{2}\left(\mathbf{x}_{1} \mid \mathbf{y}_{1}\right)=1 / d^{2} \\
h^{2}\left(\mathbf{x}_{1} \mid \mathbf{y}_{2}\right)=1 /\left(d^{2}+R_{p}^{2}\right) \\
h^{2}\left(\mathbf{x}_{2} \mid \mathbf{y}_{1}\right)=1 /\left(d^{2}+R_{p}^{2} / 4\right) .
\end{array}\right.
$$

It is now convenient to define a non-dimensional measure of the spatial resolution $\tilde{R}_{p}=R_{p} / d$ in order to derive an analytical expression that defines the resolution limit of sound pressure mapping. As a result, combining Eqs. (16) and (19) leads to

$$
\left(\beta+\frac{\beta-1}{\mathrm{SNR}}\right)+\frac{\beta}{1+\tilde{R}_{p}^{2}}-\frac{2}{1+\tilde{R}_{p}^{2} / 4}=0 .
$$

Eq. (20) can then be solved assuming that SNR $>>|\beta-1|$ as

$$
\tilde{R}_{p}=\sqrt{\frac{\sqrt{\beta^{2}-16 \beta+16}-3 \beta+4}{\beta}} .
$$

Using the $V / P$ ratio observed with the Rayleigh criterion $(\beta \approx 0.81)$ gives

$$
R_{p} \approx 2.1 d \text {. }
$$

It should be noted that the resolution expression obtained above is independent of frequency. The dependency of some techniques upon wavelength is a limitation of methodologies that rely on the interference pattern created between combinations of signals, such as traditional beamforming (see Eq. (7)). However, in the case of direct mapping, the represented result at each position is independent of other measurements since it is expressed in terms of the measured power spectrum at a particular point. Therefore, the wavelength independence is inherent to the measurement methodology. In conclusion, sound pressure mapping can be used to find uncorrelated sound sources that are separated by approximately twice the measurement distance.

\subsection{Particle velocity}

The normal component of the particle velocity can be calculated by combining Eq. (17) with Euler's equation of motion, i.e.

$$
u_{z}(\mathbf{x} \mid \mathbf{y}, t)=-\frac{1}{\mathrm{j} \omega \rho} \frac{\partial p(\mathbf{x} \mid \mathbf{y}, t)}{\partial z}=\frac{p(\mathbf{x} \mid \mathbf{y}, t)}{\rho c}\left(1+\frac{1}{\mathrm{j} k r}\right) \cos \left(\theta_{z r}\right)
$$

where $\cos \left(\theta_{z r}\right)=\hat{\mathbf{z}} \cdot \hat{\mathbf{r}}$ defining $\hat{\mathbf{z}}$ as a unitary vector perpendicular to the measurement plane $\mathbf{z}=[0,0,1]$ and $\hat{\mathbf{r}}=(\mathbf{x}-\mathbf{y}) /\|\mathbf{x}-\mathbf{y}\|$. Similar to the previous derivation, it is convenient to formulate the measured particle velocity in the 
frequency domain using the auto-power spectrum

$$
S_{u u}(\mathbf{x} \mid \mathbf{y}, \omega)=\left(\frac{|Q|}{4 \pi}\right)^{2}\left(\frac{k^{2} r^{2}+1}{r^{4}} \cos ^{2}\left(\theta_{z r}\right)\right) .
$$

The first term of Eq. (24) corresponds to the function $A^{2}$ mentioned above in the general derivation. The second term not only contains information about the quadratic decay of power with increasing distance $(1 / r)^{2}$ but also accounts for the directivity pattern of the receiving sensor $\cos ^{2}\left(\theta_{z r}\right)$ and the evanescent particle velocity field perceived close to the sound source $\left(\left(k^{2} r^{2}+1\right) / r^{4}\right)$. Recalling Eq. (16), it is now necessary to define the spatial propagation functions $h^{2}(\mathbf{x} \mid \mathbf{y})$ such as

$$
\left\{\begin{array}{l}
h^{2}\left(\mathbf{x}_{1} \mid \mathbf{y}_{1}\right)=\left(k^{2} d^{2}+1\right) / d^{4} \\
h^{2}\left(\mathbf{x}_{1} \mid \mathbf{y}_{2}\right)=k^{2} d^{2}\left(d^{2}+R_{u}^{2}\right)^{-2}+d^{2}\left(d^{2}+R_{u}^{2}\right)^{-3} \\
h^{2}\left(\mathbf{x}_{2} \mid \mathbf{y}_{1}\right)=k^{2} d^{2}\left(d^{2}+R_{u}^{2} / 4\right)^{-2}+d^{2}\left(d^{2}+R_{u}^{2} / 4\right)^{-3} .
\end{array}\right.
$$

Introducing a non-dimensional factor $\gamma=k d$ and using $\tilde{R}_{u}=R_{u} / d$, Eq. (16) then yields

$$
\left(\beta+\frac{\beta-1}{\mathrm{SNR}}\right)\left(\gamma^{2}+1\right)+\beta \frac{\gamma^{2}\left(\tilde{R}_{u}^{2}+1\right)+1}{\left(\tilde{R}_{u}^{2}+1\right)^{3}}-2 \frac{\gamma^{2}\left(\tilde{R}_{u}^{2} / 4+1\right)+1}{\left(\tilde{R}_{u}^{2} / 4+1\right)^{3}}=0 .
$$

Assuming that the signal is far greater than the noise (SNR $>>|\beta-1|)$ leads to

$$
-\frac{2}{\beta} \frac{\gamma^{2}\left(\tilde{R}_{u}^{2} / 4+1\right)+1}{\left(\tilde{R}_{u}^{2} / 4+1\right)^{3}}+\frac{\gamma^{2}\left(\tilde{R}_{u}^{2}+1\right)+1}{\left(\tilde{R}_{u}^{2}+1\right)^{3}}+\gamma^{2}+1=0 .
$$

As shown in Eq. (27), the resolution of direct particle velocity mapping has frequency dependent behaviour mainly determined by the value of $\gamma^{2}$. Although a detailed description of this expression is evaluated in the following sections, it is interesting to derive an approximate solution to understand the boundaries of the technique. Therefore,

$$
\begin{cases}-2\left(\tilde{R}_{u}^{2} / 4+1\right)^{-2}+\beta\left(1+\left(\tilde{R}_{u}^{2}+1\right)^{-2}\right)=0 & \gamma^{2}>>1 \\ -2\left(\tilde{R}_{u}^{2} / 4+1\right)^{-3}+\beta\left(1+\left(\tilde{R}_{u}^{2}+1\right)^{-3}\right)=0 & \gamma^{2}<<1\end{cases}
$$

Evaluating the last expressions numerically using $\beta \approx 0.81$, the resolution distance can be defined within the interval

$$
1.1 d<R_{u}<1.4 d \text {. }
$$

\subsection{Sound intensity}

Sound intensity describes the acoustic energy flow in a sound field [30]. The time-averaged intensity is defined as the temporal average product of sound pressure and acoustic particle velocity. In the case of a harmonic pulsating monopole in free space, the measured normal component of the sound intensity is

$$
I_{z}(\mathbf{x} \mid \mathbf{y}, \omega)=\frac{1}{2} \operatorname{Re}\left\{P(\mathbf{x} \mid \mathbf{y}, \omega) U_{z}^{*}(\mathbf{x} \mid \mathbf{y}, \omega)\right\}=\left(\frac{\rho c k^{2}|Q|^{2}}{32 \pi^{2}}\right)\left(\frac{\cos \left(\theta_{z r}\right)}{r^{2}}\right),
$$

where (.)* denotes complex conjugate. Following the established convention, the first term of Eq. (30) is analogous to the function $A^{2}$ described above. Furthermore, the second term corresponds to $h^{2}(\mathbf{x} \mid \mathbf{y})$, which comprises a power decay dependent upon the source-receiver distance $(1 / r)^{2}$, and a directivity factor $\cos \left(\theta_{z r}\right)$ introduced by the projection of the radial component of intensity on the $z$-axis. The spatial propagation functions required to derive the resolution limits of sound intensity can then be defined as

$$
\left\{\begin{array}{l}
h^{2}\left(\mathbf{x}_{1} \mid \mathbf{y}_{1}\right)=1 / d^{2} \\
h^{2}\left(\mathbf{x}_{1} \mid \mathbf{y}_{2}\right)=d /\left(d^{2}+R_{I}^{2}\right)^{3 / 2} \\
h^{2}\left(\mathbf{x}_{2} \mid \mathbf{y}_{1}\right)=8 d /\left(4 d^{2}+R_{I}^{2}\right)^{3 / 2} .
\end{array}\right.
$$

Combining previous expressions it is possible to simplify the derivation if a non-dimensional measure of the spatial resolution distance $\tilde{R}_{I}=R_{I} / d$ is introduced. The combination of the general expression together with the terms given above yields

$$
\left(\beta+\frac{\beta-1}{\mathrm{SNR}}\right)+\frac{\beta}{\left(1+\tilde{R}_{I}^{2}\right)^{3 / 2}}-\frac{16}{\left(4+\tilde{R}_{I}^{2}\right)^{3 / 2}}=0 .
$$


Assuming that the signal is far greater than the noise (SNR $>>|\beta-1|)$ leads to

$$
\frac{1}{\left(\tilde{R}_{I}^{2}+1\right)^{3 / 2}}-\frac{16}{\beta\left(\tilde{R}_{I}^{2}+4\right)^{3 / 2}}+1=0
$$

Using the peak-to-valley ratio given in the Rayleigh criterion $(\beta \approx 0.81)$ yields

$$
R_{I} \approx 1.6 d \text {. }
$$

In summary, the spatial resolution limits of direct sound mapping methods are related to the measurement distance and certain parameters that depend upon the quantity being visualize. Sound pressure mapping can be used to identify sources that are separated by more than twice the measurement distance. Sound intensity mapping provides better results since it allows distinguishing between sources that are separated by 1.6 times the measurement distance. It is however acoustic particle velocity mapping the quantity that leads to the best results, achieving a spatial resolution which varies between 1.1 and 1.4 times the measurement distance, depending on the squared wavenumber-distance product hereby denoted as $\gamma^{2}$.

\section{Numerical investigation}

A numerical investigation has been conducted to study the resolution limits proposed in the previous section. The sound field produced by two closely spaced sound sources was simulated under free field conditions. The geometry of the problem resembles the configuration shown in Fig. 2. Firstly, the levels of sound pressure, particle velocity and sound intensity at the measurement plane are shown on the left hand side of Fig. 3 for a plane located $0.05 \mathrm{~m}$ from two uncorrelated harmonic sound sources excited at $250 \mathrm{~Hz}$ and separated by $0.1 \mathrm{~m}$. Individual contributions were added using the squared amplitudes in order to fulfil the uncorrelated assumption adopted above. It should be noted that the $\mathrm{dB}$ reference varies depending on the quantity being measured $\left(p_{\text {ref }}=20 \mu \mathrm{Pa}, u_{\text {ref }}=50 \mathrm{~nm} / \mathrm{s}\right.$ and $\left.I_{\text {ref }}=1 \mathrm{pW} / \mathrm{m}^{2}\right)$.

As can be seen in this example, there are large level differences between sound pressure or sound intensity and normal particle velocity in the near field. This effect is proportional to the factor $\gamma=k r$, which is mainly caused by the evanescent energy perceived in the near-field of a sound source when measuring particle velocity. Although the evanescent velocity

(a)

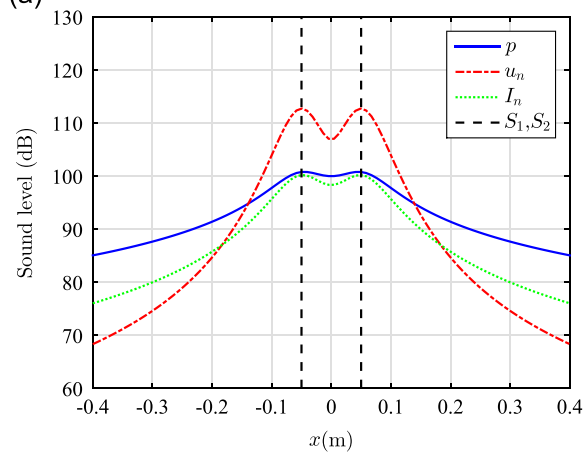

(b)

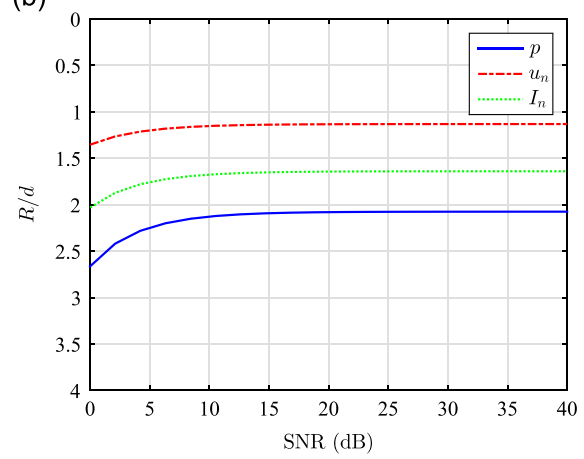

Fig. 3. Sound levels measured at a plane located $0.05 \mathrm{~m}$ from two uncorrelated harmonic sound sources excited at $250 \mathrm{~Hz}$, separated by $0.1 \mathrm{~m}$ (left) and normalized resolution achieved with the different levels of SNR (right).
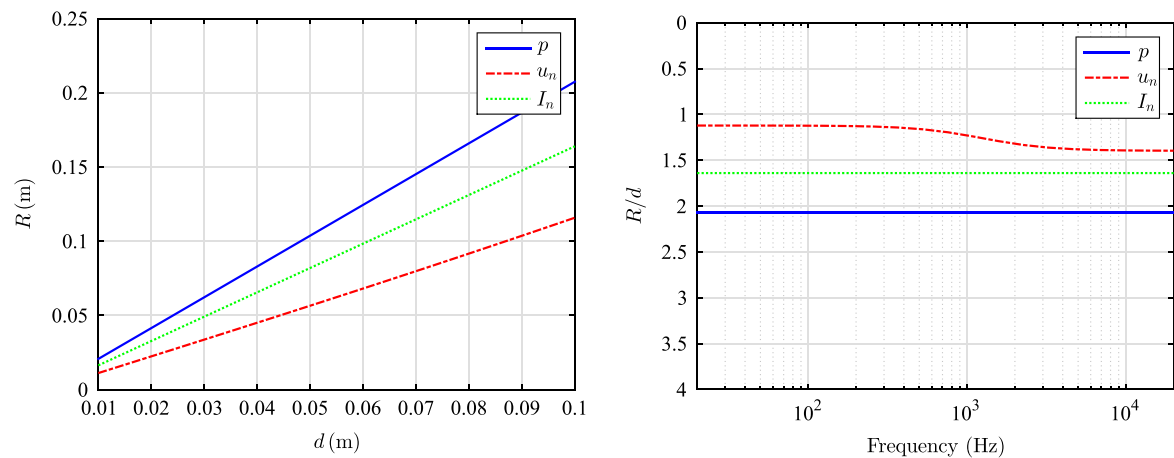

Fig. 4. Spatial resolution of direct sound mapping evaluated at $250 \mathrm{~Hz}$ (left) and normalized resolution across the audible spectra (right). 
field does not propagate far from the source, it can be beneficial for source localization purposes since it greatly enhances the signal level perceived.

The robustness of direct sound mapping is evaluated accounting for multiple SNR levels using Eqs. (20), (26) and (32). As shown on the right hand side of Fig. 3, resolution remains unaffected as long as the SNR is higher than $10 \mathrm{~dB}$. When the noise power is comparable to the source signal, the resolution distance significantly increases. If the noise can be considered mainly of acoustic nature, directivity can act as a spatial filter that enhances the SNR provided that the transducer is oriented in the normal direction, favouring the direct mapping of sound intensity, and acoustic particle velocity over sound pressure. An extended discussion of this matter is given in Appendix A.

The resolution achieved at several distances from the source plane is presented in Fig. 4. As shown, the selection of an appropriate measurement distance is crucial and determines the minimum distance at which two uncorrelated noise sources can be accurately distinguished via direct sound mapping methods. On the other hand, as illustrated on the right hand side of Fig. 4, results achieved with sound pressure or sound intensity are frequency independent, yielding a constant resolution across the spectrum. In contrast, mapping acoustic particle velocity in the acoustic near-field achieves a minimum resolvable distance that is approximately equal to the measurement distance. This near-field resolution enhancement is introduced due to the presence of an evanescence sound field which is controlled by the factor $\gamma^{2}$, as shown in Eq. (26). However, when the wavelength is shorter than the source separation $\left(\gamma^{2}>>1\right)$, the minimum resolution becomes similar to the spatial resolution achieved with direct sound intensity mapping, both significantly better than sound pressure mapping.

\section{Experimental results}

An experimental study was conducted to verify the proposed spatial resolution limits. According to the Rayleigh criterion, it is accepted that two peaks can be resolved if their $V / P$ ratio is about 0.81 or lower. In order to validate the derivations proposed in the previous section and illustrate this phenomenon, the sound field produced by two sound sources in free field conditions was studied. The separation between sources was varied whilst the measurement plane remained fixed, aiming to reproduce the expected $V / P$ ratio values used to derive the resolution limits.

The sound sources were excited with uncorrelated filtered white noise, with a centre frequency of $350 \mathrm{~Hz}$, and a bandwidth of $20 \mathrm{~Hz}$. The sources were initially driven individually in order to calibrate the system and ensure that both monopoles had approximately equal power. Measurements of sound pressure, acoustic particle velocity and sound intensity were undertaken with two $p-u$ sound intensity probes located at a plane $0.1 \mathrm{~m}$ away from the two monopole loudspeakers. A picture of the setup can be seen on the left hand side of Fig. 5 .

Test results are presented in Fig. 5 along with numerical simulations. As shown, the $V / P$ ratio calculated from measured data is very similar to the expected theoretical behaviour. Furthermore, all curves reach the value imposed by the Rayleigh criterion $(0.81)$ at the predicted points. The small discrepancy between numerical and experimental data is probably due to positioning errors during the test. This provides evidence that the conditions proposed to derive the spatial resolution limits of direct sound mapping methods can be achieved in practice. As a result, it is proven that the analytical solutions proposed can be used to predict a spatial resolution limit linked to the traditional Rayleigh criterion through the $V / P$ ratio.

\section{Conclusions}

The ability to distinguish between closely spaced acoustic sources via direct sound mapping techniques has been studied. Novel expressions describing the limits of sound pressure, normal particle velocity and sound intensity mapping have been derived, clarifying the relationship between spatial resolution, noise level and geometry. The $V / P$ ratio has been used to
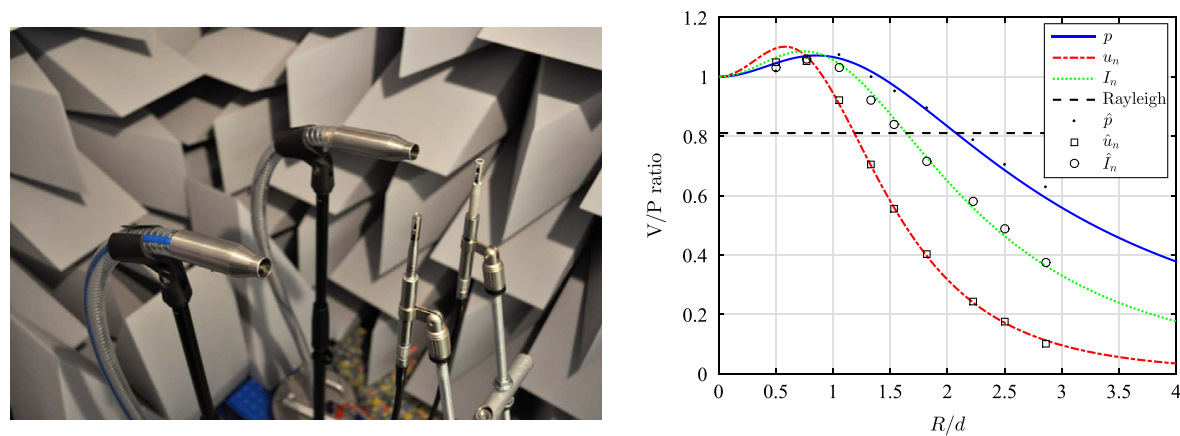

Fig. 5. Picture of the experimental setup (left) and $V / P$ ratio achieved for several source separations (right). 
quantify the quality of the most common spatial resolution criteria to establish an understanding of when two sources can be regarded as resolved.

The proposed expressions show that the resolution limits of direct sound mapping methods are linked to the source distance by factors that vary depending upon the measured quantity. Sensor directivity along with the amount of energy perceived near a sound source are the main aspects that determine the spatial resolution properties. Results obtained using particle velocity achieve the finest spatial resolution, which is furthermore enhanced in the acoustic near-field of the source. The minimum resolvable distance using this quantity varies between 1.1 and 1.4 times the measurement distance. On the other hand, the direct representation of sound intensity allows for distinguishing between sources that are separated by 1.6 times the distance to the source. In contrast, sound pressure can be used to identify sources if they are separated by approximately twice the measurement distance.

The sound field produced by two closely spaced uncorrelated monopole sound sources was studied under free field conditions via simulations and experiments. It has been shown that the predicted $V / P$ ratio can be reproduced in practice with remarkable accuracy, supporting the validity of the foundations hereby introduced. Moreover, the direct representation of sound has been proven robust against noise. It has been shown through a numerical investigation that the performance of all mapping methods remains unaffected whilst the signal-to-noise ratio is higher than $10 \mathrm{~dB}$. Below this threshold, resolution is biased, although it is still possible to differentiate between sources as long as the noise level is lower than the signal level perceived. Hence, numerical and experimental data have provided evidence that corroborate the proposed spatial resolution limits of direct sound mapping for the localization of noise sources.

\section{Acknowledgements}

The authors have discussed the substance of this paper with several of their colleagues and they would like to thank to Jaime Wild, Graciano Carrillo Pousa, Lukasz Stano and Erik-Jan Jongh for their useful input, valuable comments and kind help during the experimental test.

\section{Appendix A. Influence of sensor directivity on SNR}

The directional properties of a sensor can be linked to the SNR of a measurement as defined above. Directivity can be a useful feature for sound source localization if the sensor is aimed appropriately. The transducer can be steered towards an area of interest to maximize the sound perceived from that direction. For industrial applications, the long reverberation time and the presence of multiple disturbance sources cause the background noise to be distributed fairly homogeneously. As a result, there is an equal probability of sound waves arriving from any direction, a condition that precisely defines "diffuse" sound fields.

Free-field and pressure microphones have a sensitivity response which is ideally independent of the direction of arrival of the incident sound, i.e. they have an omni-directional directivity pattern. On the other hand, particle velocity transducers are equally sensitive to sound arriving from the front or back, but are insensitive to sound arriving from the sides, following a figure-of-eight directivity pattern.

Assuming that there are uncorrelated plane waves of equal power $\sigma_{P W}^{2}$ arriving at the sensor from all directions, the temporally averaged variance of the signal output $\sigma^{2}\left\{\overline{s^{2}(t)}\right\}$ can be calculated by integrating the individual contributions from all directions weighted by the directivity pattern $D(\theta, \phi)$, i.e.

$$
\sigma^{2}\left\{\overline{s^{2}(t)}\right\}=\int_{0}^{\pi} \int_{0}^{2 \pi} \sigma_{P W}^{2} D^{2}(\theta, \phi) \sin (\phi) \mathrm{d} \theta \mathrm{d} \phi,
$$

where $\theta$ and $\phi$ denote azimuth and elevation angles, respectively. For an omni-directional microphone with unitary gain, Eq. (35) simplifies to

$$
\sigma^{2}\left\{\overline{p^{2}(t)}\right\}=\int_{0}^{\pi} \int_{0}^{2 \pi} \sigma_{P W}^{2} \sin (\phi) \mathrm{d} \theta \mathrm{d} \phi=4 \pi \sigma_{P W}^{2}
$$

On the other hand, the figure-of-eight directivity pattern of a particle velocity transducer can be modelled using the function $\cos (\theta) \sin (\phi) / \rho c$. Substituting this term into Eq. (35) leads to

$$
\sigma^{2}\left\{\overline{u_{n}^{2}(t)}\right\}=\int_{0}^{\pi} \int_{0}^{2 \pi} \frac{\sigma_{P W}^{2}}{(\rho c)^{2}} \cos ^{2}(\theta) \sin ^{3}(\phi) \mathrm{d} \theta \mathrm{d} \phi=\frac{4 \pi \sigma_{P W}^{2}}{3(\rho c)^{2}} .
$$

The ratio between the above equations and the omni-directional result defines the effect caused by the directivity in the variance of the output signal, which corresponds to the Directivity Index (DI) when expressed in terms of level difference. 
Thus

$$
D I_{u_{n}}=10 \log \left(\frac{\sigma^{2}\left\{\overline{p^{2}(t)}\right\} / p_{\text {ref }}^{2}}{\sigma^{2}\left\{\overline{u_{n}^{2}(t)}\right\} / u_{\text {ref }}^{2}}\right) \approx 10 \log (3) \approx 4.8 .
$$

The result presented in Eq. (38) is in agreement with an alternative derivation introduced in [31]. It shows that, in a diffuse sound field, where uncorrelated wave-fronts arrive homogeneously from all directions, omnidirectional microphones capture more energy than one dimensional particle velocity transducers. Consequently, directivity acts as a spatial filter which reduces the background noise. In terms of sound level, it results in an approximately $4.8 \mathrm{~dB}$ reduction in the noise perceived when using particle velocity mapping with respect to sound pressure mapping.

In addition, sound intensity is computed as the time averaged product of sound pressure and particle velocity. As a result, the variance associated with a uni-dimensional component of sound intensity is defined as

$$
\sigma^{2}\left\{\overline{I_{n}(t)}\right\}=\sigma_{s}^{2}\left\{\overline{p(t) u_{n}(t)}\right\}=\int_{0}^{\pi} \int_{0}^{2 \pi} \sigma_{P W}^{2} \cos (\theta) \sin ^{2}(\phi) \mathrm{d} \theta \mathrm{d} \phi=0 .
$$

Sound intensity mapping is nevertheless the most robust mapping quantity under perfectly diffuse conditions, since it is theoretically unbiased in the case of isotropic acoustic noise.

\section{References}

[1] R. Beyer, Sounds of Our Times: Two Hundred Years of Acoustics, Springer Verlag GMBH, New York, 1999.

[2] Y. Kim, J. Choi, Sound Visualization and Manipulation, Wiley, Singapore, 2013.

[3] W. Gan, Acoustical Imaging: Techniques and Applications for Engineers, Wiley, Chichester, West Sussex, UK, 2012.

[4] M.R. Bai, J.-G. Ih, J. Benesty, Acoustic Array Systems: Theory, Implementation, and Application, John Wiley \& Sons, Singapore, 2013.

[5] G. Williams, Fourier Acoustics: Sound Radiation and Nearfield Acoustical Holography, Academic Press, San Diego, Calif, 1999.

[6] H. Krim, M. Viberg, Two decades of array signal processing research: the parametric approach, IEEE Signal Processing Magazine 13 (4) (1996) 67-94.

[7] F. Fahy, J. Walker, Advanced Applications in Acoustics, Noise and Vibration, CRC Press, London, 2004.

[8] J. Lanslots, F. Deblauwe, K. Janssens, Selecting sound source localization techniques for industrial applications, Sound and Vibration 44 (6) (2010) 6.

[9] M.-T. Cheng, J.A. Mann, A. Pate, Sensitivity of the wave-number domain field separation methods for scattering, The Journal of the Acoustical Society of America 99 (6) (1996) 3550-3557.

[10] C.-X. Bi, J. Stuart Bolton, An equivalent source technique for recovering the free sound field in a noisy environment, The Journal of the Acoustical Society of America 131 (2) (2012) 1260-1270.

[11] F. Fahy, Sound Intensity, E \& FN Spon, London, 1995

[12] F. Jacobsen, Sound intensity and its measurement and applications, Technical Report, Technical University of Denmark, 2011.

[13] D. Fernandez Comesaña, S. Steltenpool, G. Carrillo Pousa, H.-E. de Bree, K. Holland, Scan and paint: theory and practice of a sound field visualization method, ISRN Mechanical Engineering 2013 (241958) (2013) 1-11.

[14] K. Janssens, F. Bianciardi, D.D. Weer, Innovative sound brush measurement system for stationary sound fields, 20 th ICSV, 2013.

[15] M. Pearson, L.-A. Boudreault, A. L'espérance, F. Sgard, Sound intensity mapping with manual swiping technique, Conference of the Canadian Acoustic Association, 2010.

[16] E. Lumnitzer, Z. Farkasovska, New methods for improving of the product quality in the automotive industry, Researches and Applications in Mechanical Engineering (RAME) 2 (1) (2013) 23-29.

[17] D. Siano, M. Viscardi, M.A. Panza, Experimental acoustic measurements in far field and near field conditions: characterization of a beauty engine cover Recent Advances in Fluid Mechanics and Thermal Engineering (2014) 50-57.

[18] D. Fernandez Comesaña, Scan-Based Sound Visualisation Methods Using Sound Pressure and Particle Velocity, PhD Thesis, ISVR, University of Southampton, 2014.

[19] A.J. den Dekker, A. van den Bos, Resolution: a survey, Journal of the Optical Society of America 14 (3) (1997) 547-557.

[20] B.P. Ramsay, O.T. Koppius, E.L. Cleveland, The prism and the theory of optical resolution, Journal of the Optical Society of America 30 (September) (1940) 439-444.

[21] M.A. Lauterbach, Finding, defining and breaking the diffraction barrier in microscopy-a historical perspective, Optical Nanoscopy 1 (1) (2012) 8.

[22] D. Johnson, D. Dudgeon, Array Signal Processing: Concepts and Techniques, Prentice-Hall Signal Processing Series, P T R Prentice Hall, Englewood Cliffs, NJ, 1993.

[23] J. Christensen, J. Hald, Technical review: beamforming 1, Brüel \& Kjær Sound \& Vibration Measurement A/S, Nærum, Denmark, 2004

[24] L. Rayleigh, Xxxi. Investigations in optics, with special reference to the spectroscope, Philosophical Magazine Series 58 (49) (1879) $261-274$.

[25] W.V. Houston, A compound interferometer for fine structure work, Physical Review 29 (March) (1927) 478-484.

[26] A. Christophe, Valley to peak ratio as a measure for the separation of two chromatographic peaks, Chromatographia 4 (10) (1971) 455-458.

[27] W. Singer, M. Totzeck, H. Gross, Handbook of Optical Systems, Physical Image Formation, Vol. 2, John Wiley \& Sons, 2006, Weinheim: Wiley-VCH, $\odot 2005$

[28] Y. Kim, P. Nelson, Spatial resolution limits for the reconstruction of acoustic source strength by inverse methods, Journal of Sound and Vibration 265 (3) (2003) 583-608.

[29] E.G. Williams, J.D. Maynard, Holography imaging without the wavelength resolution limit, Physical Review Letters 45 (August) (1980) 554-557.

[30] F. Jacobsen, P. Juhl, Fundamentals of General Linear Acoustics, Wiley, Chichester, West Sussex, United Kingdom, 2013.

[31] F. Jacobsen, D. tekniske højskole. Laboratoriet for akustik, The diffuse sound field: statistical considerations concerning the reverberant field in the steady state, Lyngby, Technical University of Denmark, Acoustics Laboratory Report, 1979. 